\title{
PAR-2 expression in the gingival crevicular fluid reflects chronic periodontitis severity
}

\author{
Henrique FUKUSHIMA(a) \\ Vanessa Tubero Euzebio ALVES(a) \\ Verônica Franco de CARVALHO(a) \\ Lucas Macedo Batitucci \\ AMBRÓSIO(a) \\ Rosangela Aparecida dos Santos \\ EICHLER $^{(b)}$ \\ Maria Helena Catelli de \\ CARVALHO(b) \\ Luciana SARAIVA(a) \\ Marinella HOLZHAUSEN(a) \\ (a) Universidade de São Paulo - USP, School of \\ Dentistry, Department of Stomatology, São \\ Paulo, SP, Brazil. \\ (b)Universidade de São Paulo - USP, \\ Biomedical Institute, Division of \\ Pharmacology, São Paulo, SP, Brazil.
}

Declaration of Interests: The authors certify that they have no commercial or associative interest that represents a conflict of interest in connection with the manuscript.

Corresponding Author:

Marinella Holzhausen

E-mail: marinella@usp.br

http://doi.org/10.1590/1807-3107BOR-2017.vol31.0016

Submitted: June 01, 2016

Accepted for publication: Nov 21, 2016

Last revision: Dec 08, 2016

\begin{abstract}
Recent studies investigating protease-activated receptor type 2 (PAR-2) suggest an association between the receptor and periodontal inflammation. It is known that gingipain, a bacterial protease secreted by the important periodontopathogen Porphyromonas gingivalis can activate PAR-2. Previous studies by our group found that PAR-2 is overexpressed in the gingival crevicular fluid (GCF) of patients with moderate chronic periodontitis (MP). The present study aimed at evaluating whether PAR-2 expression is associated with chronic periodontitis severity. GCF samples and clinical parameters, including plaque and bleeding on probing indices, probing pocket depth and clinical attachment level, were collected from the control group ( $n=19)$ at baseline, and from MP patients $(n=19)$ and severe chronic periodontitis $(\mathrm{SP})(\mathrm{n}=19)$ patients before and 6 weeks after periodontal non-surgical treatment. PAR-2 and gingipain messenger RNA (mRNA) in the GCF of 4 periodontal sites per patient were evaluated by Reverse Transcription Polymerase Chain Reaction (RT-qPCR). PAR-2 and gingipain expressions were greater in periodontitis patients than in control group patients. In addition, the SP group presented increased PAR-2 and gingipain mRNA levels, compared with the MP group. Furthermore, periodontal treatment significantly reduced $(p<0.05)$ PAR-2 expression in patients with periodontitis. In conclusion, PAR-2 is associated with chronic periodontitis severity and with gingipain levels in the periodontal pocket, thus suggesting that PAR-2 expression in the GCF reflects the severity of destruction during periodontal infection.
\end{abstract}

Keywords: Chronic Periodontitis; Receptor PAR-2; Porphyromonas gingivalis; Argingipain, Porphyromonas Gingivalis.

\section{Introduction}

Protease activated receptors (PARs) belong to a family of G-protein seventransmembrane domain receptors. Activation of these receptors occurs through proteolytic cleavage of the extracellular domain, which generates a new $\mathrm{N}$-terminal ligand that binds to the receptor itself, triggering intracellular signaling. To date, four types of PARs have been identified: PAR-1, $-2,-3$ and -4 . These receptors have similar mechanisms of activation; however, they may have different tissue locations and functions, and can be activated by different proteases. ${ }^{1,2}$ 
PAR-2, activated by trypsin, tryptase and coagulation factors VIIa and Xa, actively participates in inflammatory processes, such as neutrophil rolling, adhesion and extravasation of leukocytes, increased vascular permeability, edema, granulocyte recruitment and degranulation of mast cells. ${ }^{3,4,5,6}$ Recently, some studies have suggested the involvement of PAR-2 in periodontal inflammation. ${ }^{78,9,10,11,12,13,14}$

Lourbakos et al., ${ }^{7}$ reported that the bacterial cysteine proteases produced by Porphyromonas gingivalis $(P g)$, gingipains, can activate PAR-2 in oral epithelial cells and induce the secretion of interleukin 6 (IL-6), a proinflammatory cytokine that stimulates the release of osteoclasts and bone resorption. In a previous study, PAR-2 activation by a selective agonist led to periodontal inflammation and alveolar bone loss in rats through a mechanism involving the release of prostaglandins and activation of matrix metalloproteinases (MMPs). ${ }^{10}$ In addition, human studies have found that patients with moderate chronic periodontitis show a higher gingival crevicular fluid (GCF) PAR-2 expression than healthy subjects. ${ }^{12}$ Thus, the studies in the literature strongly suggest an association of PAR-2 with inflammation and alveolar bone loss in chronic periodontitis. Accordingly, a recent study by Euzebio Alves et al., 2013, ${ }^{14}$ observed that periodontal treatment significantly reduced GCF PAR-2 expression in patients with moderate chronic periodontitis.

Interestingly, Euzébio-Alves et al., ${ }^{14}$ found that the reduced expression of PAR-2 after non-surgical periodontal treatment was correlated with reduced gingipain expression, thus suggesting that increased PAR-2 expression in diseased patients is not a constitutive feature of the individual. However, to date, it has not been determined whether the severity of the disease is associated with increased PAR-2 expression. We hypothesized that PAR-2 expression is higher in the GCF of patients with severe versus moderate chronic periodontitis. Thus, the aim of this study was to verify whether PAR-2 expression in the GCF is associated to the intensity of the periodontal destruction.

\section{Methodology}

\section{Patient selection and study protocol}

Patients were recruited at the dental clinics of the School of Dentistry, University of Sao Paulo (FOUSP). After being advised of the nature and objectives of the study, the patients signed an informed consent form approved by the ethics committee of the School of Dentistry, University of São Paulo, Brazil (FR-337902, Protocol 106/2010).

The subjects were enrolled consecutively, based on the inclusion and exclusion criteria. Patients of both genders aged 25 to 60 years, with good general health, were included. In this evaluation, a medical history and a clinical examination were performed.

This study included 19 patients with generalized severe chronic periodontitis (SP group) - presence of periodontal attachment loss $\geq 5 \mathrm{~mm}$ in over $30 \%$ of the remaining teeth, ${ }_{1}^{15} 19$ patients with generalized moderate chronic periodontitis (MP group) - presence of periodontal attachment loss of 3-4 $\mathrm{mm}$ in over $30 \%$ of the remaining teeth, ${ }^{15}$ and 19 periodontally healthy patients (C group) - absence of clinical signs of inflammation and/or attachment loss ${ }^{15}$. Exclusion criteria were the following: need for prophylactic antibiotic therapy; ${ }^{16}$ diabetic, smoker, immunocompromised, pregnant or lactating patients; use of drugs such as phenytoin and cyclosporine or calcium channel blockers such as nifedipine; previous periodontal treatment and/or antibiotic use in the last six months; presence of severe occlusal discrepancies and/or use of orthodontic appliances; abutment teeth or prosthesis retainers, teeth with grade II and III mobility, and teeth with endodontic lesions.

The clinical parameters analyzed were: visible plaque index (PI) ${ }_{1}^{17}$ bleeding on probing (BP) index, ${ }^{18}$ probing pocket depth (PPD) ${ }^{19}$ and clinical attachment level (CAL), ${ }^{19}$ and were determined by using a manual probe (PCPUNC 15-6, Hu-Friedy, Chicago, IL, USA). The clinical evaluation was performed by two trained and calibrated examiners. Calibration was done before performing the baseline exams, and again before performing the re-evaluation exams. The reproducibility of intra- and interexaminer was verified by the intraclass correlation coefficient for 
continuous variables (PPD and CAL) and the kappa coefficient (0.92).

Patients with moderate and severe chronic periodontitis received non-surgical periodontal treatment, which consisted of oral hygiene instructions, scaling and root planing with manual instruments (Gracey curettes, Hu-Friedy, Chicago, IL, USA) and sonic devices (Minipiezon ${ }^{2}$, EMS, Nyon, Switzerland), and clinical integration (temporary restoration of cavities, extraction of hopeless teeth, and elimination of iatrogenic restorative factors, if necessary). Healthy patients received prophylaxis and oral hygiene instructions for plaque control.

The treatment sessions were defined by the characteristics and conditions of each patient. Forty-five days after the end of the non-surgical periodontal treatment, all the patients were submitted to weekly professional plaque control (oral hygiene reinforcement, supragingival scaling and dental polish) until the time of the reassessment appointment. During re-evaluation, the same initial clinical parameters as those described above were evaluated.

\section{Gingival crevicular fluid (GCF) sample collection}

The deepest periodontal site per quadrant was selected for GCF sampling in the MP group $(4 \mathrm{~mm} \leq \mathrm{PPD} \leq 5 \mathrm{~mm})$ and in the SP group (PPD $>5 \mathrm{~mm}$ ). After the patients received periodontal therapy, GCF was collected from the same 4 sites. One healthy periodontal site per quadrant, with absence of inflammation and/or attachment loss, was sampled in the control group. After removal of the supragingival plaque with periodontal curettes, the periodontal sites were isolated using sterile cotton rolls, and a periopaper strip (Periopaper Collection Strip, Oraflow, Plainview, NY, USA) was introduced in the gingival sulcus/periodontal pocket until a mild resistance was felt, and was then left in place for 30 seconds. After removal, the volume of GCF was determined using an appropriate device (Periotron 6000, IDE Interstate, Amityville, NY). The periopaper was placed in plastic tubes containing $400 \mu \mathrm{l}$ of phosphate buffer solution (pH 7.4). After being vortexed for 30 seconds, the samples were centrifuged in a refrigerated microcentrifuge for 10 minutes at $6000 \mathrm{~g}$, and the precipitate was stored at $80^{\circ} \mathrm{C}$ in tubes containing $1 \mathrm{ml}$ of RNA stabilizing reagent, Tri-Reagent (Sigma, St. Louis, USA) until further analysis.

\section{PAR-2 and gingipain mRNA expression}

The PAR-2 mRNA expression present in the GCF samples was evaluated by Reverse Transcription Polymerase Chain Reaction (RT-qPCR). Total RNA (tRNA) was obtained by homogenization of the GCF using Trizol (guanidine isothiocyanate) (GITC) solubilized in a phenol solution, according to the manufacturer (Invitrogen Brasil, LTDA., São Paulo, Brazil). Quantification of tRNA was determined by resuspending the pellet in $12 \mu \mathrm{l}$ of $0.01 \%$ inactivated diethylpyrocarbonate (DEPC) water, and the readings were performed with $1 \mu l$ of the sample in duplicate. After quantification, the remaining $10 \mu \mathrm{l}$ of the tRNA was used to synthesize the cDNA of the first strand, using SuperScript II and RNaseOUT. The GoTaq qPCR Master Mix (Promega, Madison, WI, USA) and the specific oligonucleotides for PAR-2 and Arg-gingipain B (RgpB), obtained from the GenBank (http://www. Ncbi.nlm.nih.gov/tools/primer-Blast) (Table 1), were used for the analysis. GAPDH gene expression was used as a reference gene for PAR-2 expression analysis, and $16 \mathrm{~S}$ ribosomal RNA was used as a constitutive bacterial gene for gingipain analysis. The reactions were performed with the Corbett Research system (Corbett Life Sciences, Sydney, Australia). The oligonucleotides were purchased from Invitrogen Co., San Diego, CA.

\section{Statistical analysis}

A priori sample size calculation revealed a requirement of 16 patients for the three groups (control, MP, and SP), assuming a standard deviation of 1.5 and an anticipated PAR-2 genetic expression difference of 2 , with a significance level of $5 \%$ and a statistical power of $90 \%$. Thus, considering a loss to follow-up of $15 \%, 19$ patients were included per group.

The normality of the distribution of each variable was assessed by the Shapiro-Wilk test. The differences between the two chronic periodontitis groups (MP and SP) and the control group were analyzed by one-way ANOVA. In case of significant differences between these groups, posthoc two-group comparisons were assessed 
Table 1. Sequence of primers used for cDNA amplification.

\begin{tabular}{lccc}
\hline Variable & Forward (F) and reverse (R) & Gene bank access number & Fragment size \\
\hline GAPDH (F) & 5'-TGGTATCGTGGAAGGACTCATGAC-3' & NM_002046 & $80 \mathrm{pb}$ \\
GAPDH (R) & 5'- ATGCCAGTGAGCTTCCCGTTCAGC-3' & \\
PAR-2 (F) & 5'-TGCTAGCAGCCTCTCTCTCC -3' & NM_053897.2 & $92 \mathrm{pb}$ \\
PAR-2 (R) & 5'-TGTGCCATCAACCTTACCAA -3' & \\
Gingipain (F) & 5'-CCTACGTGTACGGACAGAGCTATA-3' & NC_010729 & 70pb \\
Gingipain (R) & 5'-AGGATCGCTCAGCGTAGCATT-3' & & $86 \mathrm{pb}$ \\
16S Ribosomal (F) & 5'-TCGGTATTGAGGAAGGTTGG-3' & NC_015571 \\
16S Ribosomal (R) & 5'-CTGCTGGCACGGAGTTAG-3' & \\
\hline
\end{tabular}

with the Tukey-Kramer test. Comparisons between baseline and post-treatment within the chronic periodontitis groups were analyzed with a paired ttest. Differences in the ration of males to females, and the incidence of BP among the groups, were analyzed with a $\mathrm{X}^{2}$ test.

The Pearson correlation analysis was performed with the parameters of individual sampled sites, and was used to test the associations between PAR-2 gene expression and PPD, CAL and gingipain gene expression. The analyses and graphics were done using the GraphPad Prism statistical software (GraphPad Software Inc., La Jolla, CA, USA). A value of $p<0.05$ was considered statistically significant. Data were expressed as means \pm standard deviation.

\section{Results}

Nineteen patients were selected for each group, based on the inclusion and exclusion criteria. Three patients from the SP group were excluded during the study for not attending the appointments.

The results of the demographic analysis showed similarity in gender distribution, nine females and ten males, aged between 31 and 58 years (Table 2).

\section{Clinical parameters}

Statistical analysis showed significant differences $(p<0.05)$ for all the periodontal clinical parameters for both the chronic periodontitis patients (MP and SP) and the control patients. PPD and PI parameters
Table 2. Demographic data

\begin{tabular}{lccc}
\hline Variable & Control group & $\begin{array}{c}\text { Moderate } \\
\text { periodontitis }\end{array}$ & $\begin{array}{c}\text { Severe } \\
\text { periodontitis }\end{array}$ \\
\hline $\mathrm{N}$ & 19 & 19 & 16 \\
$\begin{array}{l}\text { Gender } \\
\text { Age } \\
\text { (years) }\end{array}$ & $10 \mathrm{M} / 9 \mathrm{~F}$ & $10 \mathrm{M} / 9 \mathrm{~F}$ & $9 \mathrm{M} / 7 \mathrm{H}$ \\
\hline
\end{tabular}

Differences in the ratio of males to females were analyzed by a $X^{2}$ test. The differences between the control group and the chronic periodontitis groups were analyzed by one-way ANOVA followed by post-hoc two-group comparisons assessed by the Tukey-Kramer test. $\mathrm{N}$ : number of patients; $\mathrm{M}$ : male; F: female.

were statistically higher in the SP than the MP group $(\mathrm{p}<0.05)$.

Non-surgical periodontal treatment statistically reduced the PI, BP and PPD parameters $(\mathrm{p}<0.05)$ in both the MP and the SP groups (Table 3 ). In addition, periodontal treatment led to a significant improvement in CAL, only in the SP group ( $\mathrm{p}<0.05)$.

Table 3 shows that the periodontal clinical parameters (PPD, CAL, BP and PI) of the sample sites were statistically higher $(p<0.05)$ in the periodontitis groups than the control group. Treated periodontal sites from the chronic periodontitis groups (MP and SP) showed significant reductions $(\mathrm{p}<0.05)$ in the PPD, CAL, BP and PI parameters (Table 4).

\section{PAR-2 expression in the GCF}

Severe chronic periodontitis patients presented a higher PAR-2 expression $(p<0.05)$ than either 
Table 3. Mean and standard deviation of clinical periodontal parameters of the control and the chronic periodontitis groups at baseline and after nonsurgical periodontal therapy.

\begin{tabular}{lccccc}
\hline Variable & Control group & $\begin{array}{c}\text { Moderate periodontitis } \\
\text { (baseline) }\end{array}$ & $\begin{array}{c}\text { Moderate periodontitis } \\
\text { (after) }\end{array}$ & $\begin{array}{c}\text { Severe periodontitis } \\
\text { (baseline) }\end{array}$ & $\begin{array}{c}\text { Severe periodontitis } \\
\text { (after) }\end{array}$ \\
\hline PPD (mm) & $1.84 \pm 0.28$ & $2.79 \pm 0.41^{a}$ & $2.22 \pm 0.29^{a, b}$ & $3.30 \pm 0.7^{a, b, c}$ & $2.33 \pm 0.31^{a, b, d}$ \\
CAL (mm) & $2.11 \pm 0.37$ & $3.51 \pm 0.57^{a}$ & $3.15 \pm 0.65^{a}$ & $4.14 \pm 1.03^{a, c}$ & $3.26 \pm 0.95^{a, d}$ \\
BP (\%) & $3.33 \pm 2.89$ & $55.87 \pm 20.83^{a}$ & $16.47 \pm 27.00^{b}$ & $42.14 \pm 20.09^{a, c}$ & $11.14 \pm 4.45^{b, d}$ \\
PI (\%) & $11.88 \pm 10.78$ & $86.85 \pm 14.95^{a}$ & $25.91 \pm 14.95^{b}$ & $63.33 \pm 23.00^{a, b, c}$ & $15.47 \pm 7.65^{b, c, d}$ \\
\hline
\end{tabular}

PPD: probing pocket depth; CAL: clinical attachment level; BP: bleeding on probing; PI: plaque index; a: statistically different $(p<0.05)$ from the control group; b: statistically different $(p<0.05)$ from the moderate periodontitis group at baseline; c: statistically different $(p<0.05)$ from the moderate periodontitis group after periodontal therapy; $d$ : statistically different $(p<0.05)$ from the severe periodontitis group at baseline.

Table 4. Mean and standard deviation of clinical periodontal parameters of the periodontal sample sites from the control and the chronic periodontitis groups at baseline and after nonsurgical periodontal therapy.

\begin{tabular}{lccccc}
\hline Variable & Control group & $\begin{array}{c}\text { Moderate periodontitis } \\
\text { (baseline) }\end{array}$ & $\begin{array}{c}\text { Moderate periodontitis } \\
\text { (after) }\end{array}$ & $\begin{array}{c}\text { Severe periodontitis } \\
\text { (baseline) }\end{array}$ & $\begin{array}{c}\text { Severe periodontitis } \\
\text { (after) }\end{array}$ \\
\hline PPD (mm) & $2.15 \pm 0.47$ & $5.05 \pm 0.81^{a}$ & $3.19 \pm 0.87^{a, b}$ & $6.26 \pm 1.17^{a, b, c}$ & $3.13 \pm 0.72^{a, b, d}$ \\
CAL (mm) & $2.24 \pm 0.53$ & $6.13 \pm 1.15^{a}$ & $4.39 \pm 1.15^{a, b}$ & $7.01 \pm 1.44^{a}$ & $4.48 \pm 1.05^{a, d}$ \\
BP (\%) & $0.00 \pm 0.00$ & $100.00 \pm 0.00^{a}$ & $26.32 \pm 35.82^{a, b}$ & $73.68 \pm 28.23^{a, c}$ & $21.31 \pm 16.90^{a, d}$ \\
PI (\%) & $2.21 \pm 6.61$ & $91.55 \pm 13.95^{a}$ & $33.40 \pm 32.07^{a, b}$ & $49.10 \pm 30.50^{a, b, c}$ & $7.73 \pm 13.45^{a, b, d}$ \\
\hline
\end{tabular}

PPD: probing pocket depth; CAL: clinical attachment level; BP: bleeding on probing; PI: plaque index; a: statistically different $(p<0.05)$ from the control group; $b$ : statistically different $(p<0.05)$ from the moderate periodontitis group at baseline; c: statistically different $(p<0.05)$ from the moderate periodontitis group after therapy; $d$ : statistically different $(p<0.05)$ from the severe periodontitis group at baseline.

periodontally health patients (threefold increase) and or moderate chronic periodontitis patients (twofold increase). Non-surgical periodontal treatment significantly reduced $(\mathrm{p}<0.05)$ PAR-2 gene expression in the periodontitis groups (both MP and SP) (Figure 1).

There was a positive correlation between the PAR-2 expression in the GCF and the mean PPD values $(r=0.64 ; p<0.01)$ and the CAL values $(r=0.40 ; p<0.01)$

\section{Gingipain expression in the GCF}

Gingipain mRNA expression was significantly higher $(\mathrm{p}<0.05)$ in the chronic periodontitis (both MP and SP) patients than the control patients. Moreover, the SP group showed a higher gingipain expression than the MP group $(\mathrm{p}<0.05)$ (Figure 2).
After periodontal therapy, gingipain expression was decreased in both groups (MP and SP) compared to baseline $(\mathrm{p}<0.05)$. There was a positive correlation between gingipain expression and PAR-2 expression in the GCF $(r=0.41 ; p<0.01)$ (Figure 2).

\section{Discussion}

The present study examined the expression of PAR-2 in patients with different severity of chronic periodontitis. The main finding of this study was the positive correlation between periodontal disease severity and PAR-2 expression in the GCF. Patients with severe chronic periodontitis showed a twofold higher PAR-2 expression than patients with moderate chronic periodontitis, and threefold higher expression than periodontally healthy patients. 


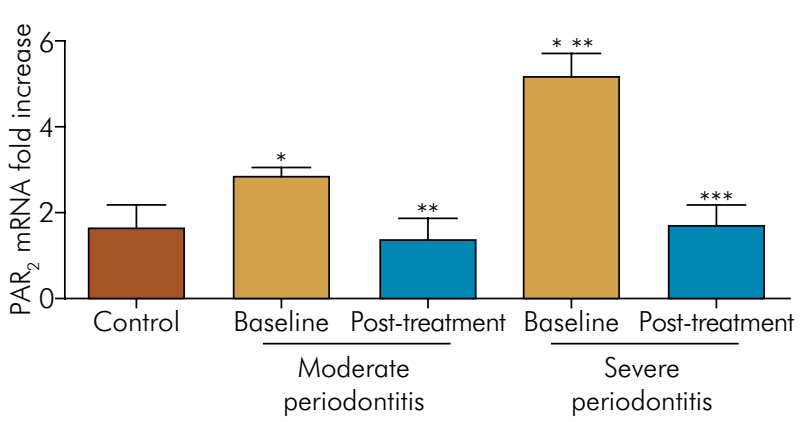

* Statistically different $(p<0.05)$ from the control group;

**Statistically different $(p<0.05)$ from the moderate chronic periodontitis group at baseline; ${ }^{* * *}$ Statistically different $(p<0.05)$ from the severe chronic periodontitis group at baseline.

Figure 1. Mean and standard deviation of PAR-2 mRNA in the GCF of the control group and groups with chronic periodontitis at baseline and after non-surgical periodontal therapy.

Interestingly, it was previously demonstrated that the genetic upregulation of PAR-2 reflects its translated active protein levels in chronic periodontitis sites. ${ }^{14}$ In fact, there is a very strong positive correlation $(\mathrm{r}=0.8935)$ between PAR-2 mRNA expression and PAR-2 protein levels in the GCF.

The present study showed that PAR-2 expression was significantly associated with clinical measurements of disease severity (e.g., pocket depth and attachment level). In addition, nonsurgical periodontal treatment reduced PAR-2 expression in patients with chronic periodontitis, and this reduction was associated with improved clinical parameters. This corroborates the study by Euzébio-Alves et al., ${ }^{14}$ which also verified the reduction in proinflammatory cytokines, such as IL-6, IL-8, TNF-a, MMP-2, MMP-8, HGF, and VEGF.

Since the PAR-2 expression in the present study was analyzed in the GCF samples, it may reflect the expression of the cells present in this environment, such as lymphocytes, neutrophils, mast cells and oral epithelial cells. ${ }^{1,2,7,8,9,20,21}$ It is known that activation of the PAR-2 receptor in these cells could lead to the secretion of several proinflammatory cytokines, such as IL-1, IL-1 $\beta$, IL-6, IL-8, IFN- $\gamma$, PGE2, and MMP 9. ${ }^{8,9,22,23,24}$ Interestingly, the study by Euzébio-Alves et al., ${ }^{14}$ clearly demonstrated that epithelial cells and leukocytes present in the GCF express PAR-2, and that the presence of the

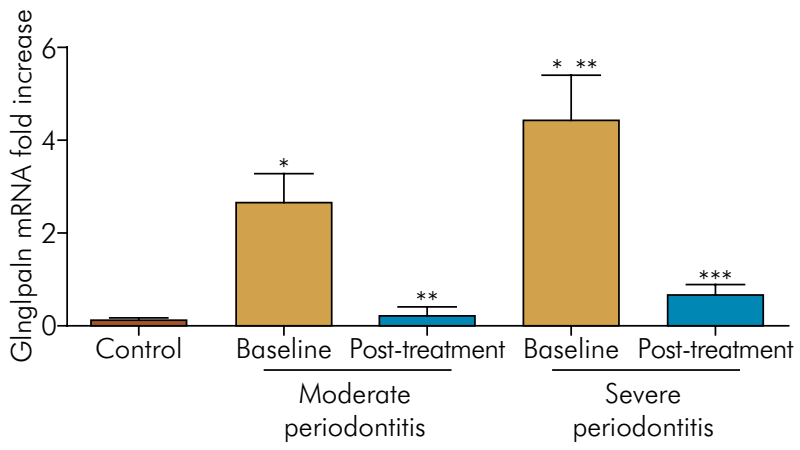

*Statistically different $(p<0.05)$ from the control group;

**Statistically different $(p<0.05)$ from the moderate chronic periodontitis group at baseline; ${ }^{* * *}$ Statistically different $(p<0.05)$ from the severe chronic periodontitis group at baseline.

Figure 2. Mean and standard deviation of gingipain mRNA in the GCF of the control group and groups with chronic periodontitis at baseline and after non-surgical periodontal therapy.

potential activators, gingipains and neutrophil serine proteinase 3 , and SLPI and elafin serine protease inhibitors all influence its expression.

The present study also demonstrated that PAR-2 expression in the GCF reflects the presence of infection by $P g$, since there was a positive correlation between the expression of PAR-2 and the expression of the bacterial protease gingipain, which plays a known role in PAR-2 activation. ${ }^{7,89}$ A previous study by Fagundes et al., ${ }^{13}$ also found a positive association between the greater presence of $P g$-positive periodontal sites and increased PAR-2 expression. Similarly, other studies associated the presence of microorganisms with increased PAR-2 expression: Helicobacter pylori in human gastric epithelial cells, ${ }^{25}$ Salmonella typhimurium in mouse neutrophils of peripheral blood, ${ }^{26}$ influenza A/PR-8/34 virus in the epithelial cell airways of mice, ${ }^{27}$ and Cryptosporidium parvum in human ileocecal epithelial cells. $^{28}$

It should be pointed out that the analyses performed in the present study did not aim to demystify the cascades of activation in which the PAR-2 is involved, or any other interaction that occurs between gingipain and the host and/or other factors involved in periodontal destruction. Rather, the scope of this study was to evaluate the association between disease severity and PAR-2 expression. Future studies may clarify the inflammatory mechanisms associated with the activation of PAR-2 in periodontal disease, 
using pharmaceutical agents that could modulate the receptor.

\section{Conclusion}

In conclusion, the present study demonstrated that PAR-2 mRNA in the GCF reflects the severity of periodontal destruction. The data reported in this study provide a basis for future prospective longitudinal studies on the possible relevance of PAR-2 as a prognostic marker in periodontitis, and for a better understanding of disease immune-inflammatory processes, as a prerequisite for designing future treatment strategies.

\section{Acknowledgments}

This work was supported by the Sao Paulo State Research Foundation (FAPESP, Sao Paulo, SP, Brazil), Research Grant \#2010/16605-0. HF and VTEA receive a scholarship from the Coordination for the Improvement of Upper Education Personnel (CAPES, Brasilia, DF, Brazil) and FAPESP, respectively.

\section{References}

1. Böhm SK, Kong W, Bromme D, Smeekens SP, Anderson $\mathrm{AD}$, Connolly A et al. Molecular cloning, expression and potential functions of the human proteinase-activated receptor-2. Biochem J. 1996;314(3):1009-16. http://doi.org/10.1042/bj3141009

2. Ossovskaya VS, Bunnett NW. Protease activated receptor: contribution to physiology and disease. Physiol Rev. 2004;84(2):579-621. http://doi.org/10.1152/physrev.00028.2003

3. Molino M, Barnathan ES, Numerof R, Clark J, Dreyer M, Cumashi A et al. Interactions of mast cell tryptase with thrombin receptors and PAR-2. J Biol Chem. 1997;272(7):4043-9. http://doi.org/10.1074/jbc.272.7.4043

4. Corvera CU, Dery O, McConalogue K, Böhm SK, Khitin LM, Caughey GH et al. Mast cell tryptase regulates rat colonic myocytes through proteinase-activated receptor 2. J Clin Invest. 1997;100(6):1383-93. http://doi.org/10.1172/JCI119658

5. Vergnolle N, Hollenberg MD, Wallace JL. Pro- and anti-inflammatory actions of thrombin: a distinct role for proteinase-activated receptor-1 (PAR-1). Br J Pharm. 1999;126(5):1262-8. http://doi.org/10.1038/sj.bjp.0702408

6. Vergnolle N, Hollenberg MD, Sharkey KA, Wallace JL. Characterization of the inflammatory response to proteinase-activated receptor-2(PAR2)-activating peptides in the rat paw. Br J Pharm. 1999;127:1083-90. http://doi.org/10.1038/sj.bjp.0702634

7. Lourbakos A, Chinni C, Thompson P, Potempa J, Travis J, Mackie EJ et al. Cleavage and activation of proteinase-activated receptor-2 on human neutrophils by gingipain- $\mathrm{R}$ from Porphyromonas gingivalis. FEBS Lett. 1998;435(1):45-8. http://doi.org/10.1016/S0014-5793(98)01036-9

8. Lourbakos A, Potempa J, Travis J, D’Andrea MR, Andrade-Gordon P, Santulli R et al.
Arginine-specific protease from Porphyromonas gingivalis activates protease-activated receptors on human oral epithelial cells and induces interleukin-6 secretion. Infect Immun. 2001;69(8):5121-30. http://doi.org/10.1128/IAI.69.8.5121-5130.2001

9. Lourbakos A, Yuan YP, Jenkins AL, Travis J, Andrade-Gordon P, Santulli R et al. Activation of protease-activated receptors by gingipains from Porphyromonas gingivalis leads to platelet aggregation: a new trait in microbial pathogenicity. Blood. 2001;97(12):3790-7. http://doi.org/10.1182/blood.V97.12.3790

10. Holzhausen M, Spolidorio LC, Vergnolle N. Protease-activated receptor-2(PAR-2) agonist causes periodontitis in rats. J Dent Res. 2005;84(2):154-9. http://doi.org/10.1177/154405910508400209

11. Holzhausen M, Spolidorio LC, Ellen RP, Jobin $\mathrm{MC}$, Steinhoff M, Andrade-Gordon P et al. Protease-activated receptor-2 activation: a major role I the pathogenesis of Porphyromonas gingivalis infection. Am J Pathol. 2006;168(4):1189-99. http://doi.org/10.2353/ajpath.2006.050658

12. Holzhausen M, Cortelli JR, Silva VA, Franco GCN, Cortelli SC, Vergnolle N. Protease-activated receptor-2 (PAR-2) in human periodontitis. J Dent Res. 2010;89(9):948-53. http://doi.org/10.1177/0022034510373765

13. Fagundes JAG, Monoo LD, Alves VTE, Pannuti CM, Cortelli SC, Cortelli JR et al. Porphyromonas gingivalis is associated with protease-activated receptor-2 upregulation in chronic periodontitis. J Periodontol. 2011;82(11):1596-601. http://doi.org/10.1902/jop.2011.110073

14. Euzébio-Alves VT, Silva HAB, França BN, Eichler RS, Saraiva L, Carvalho MHC et al. Periodontal treatment downregulates protease-activated receptor- 2 in human gingival crevicular fluid cells. Infect Immun. 2013;81(12):4399-407. http://doi.org/10.1128/IAI.01107-13 
15. Armitage GC. Development of a classification system for periodontal diseases and conditions. Ann Periodontol. 1999;4(1):1-6. http://doi.org/10.1902/annals.1999.4.1.1

16. Wilson W. Taubert KA, Gewitz M, Lockhart PB, Baddour LM, Levison M et al. Prevention of infective endocarditis: guidelines from the American Heart Association. J Am Dent Assoc. 2007;138(6):739-60. http://doi.org/10.14219/jada.archive.2007.0262

17. Ainamo J, Bay I. Problems and proposals for recording gingivitis and plaque. Int Dent J. 1975;25(4):229-35.

18. Greenstein $\mathrm{G}$. The role of bleeding upon probing in the diagnosis of periodontal disease. J Periodontol. 1984;55(12):684-8. http://doi.org/10.1902/jop.1984.55.12.684

19. Glavind L, Loe H. Errors in the clinical assessment on periodontal destruction. J Periodontal Res. 1967;2(3):180-4. http://doi.org/10.1111/j.1600-0765.1967.tb01887.x

20. Nystedt S, Ramakrishnan V, Sundelin J. The proteinase-activated receptor 2 is induced by inflammatory mediators in human endothelial cells: comparison with the thrombin receptor. J Biol Chem. 1996;271(25):14910-5. http://doi.org/10.1074/jbc.271.25.14910

21. Abraham LA, Chinni C, Jenkins AL, Lourbakos A, Ally N, Pike RN et al. Expression of protease-activated receptor-2 by osteoblasts. Bone. 2000;26(1):7-14. http://doi.org/10.1016/S8756-3282(99)00237-9

22. Shpacovitch VM, Varga G, Strey A, Gunzer M, Mooren F, Buddenkotte J et al. Agonists of proteinase-activated receptor-2 modulate human neutrophil cytokine secretion, expression of cell adhesion molecules, and migration within 3-D collagen lattices. J Leukoc Biol. 2004;76(2):388-98. http://doi.org/10.1189/jlb.0503221
23. Lee HM, Kim HY, Kang HJ, Woo JS, Chae SW, Lee SH et al. Up-regulation of protease-activated receptor 2 in allergic rhinitis. Ann Otol Rhinol Laryngol. 2007;116(7):554-8. http://doi.org/10.1177/000348940711600712

24. Moraes TJ, Martin R, Plumb JD, Vachon E, Cameron CM, Danesh A et al. Role of PAR-2 in murine pulmonary pseudomonal infection. Am J Physiol Lung Cell Mol Physiol. 2007;294(2):L368-77. http://doi.org/10.1152/ajplung.00036.2007

25. Kajikawa H, Yoshida N, Katada K, Hirayama F, Handa O, Kokura S et al. Helicobacter pylori activates gastric epithelial cells to produce interleukin-8 via protease-activated receptor 2. Digestion. 2008;76(3-4):248-55. http://doi.org/10.1159/000113041

26. St-Onge M, Lagarde S, Laflamme C, Rollet-Labelle E, Marois L, Naccache PH et al. Proteinase-activated receptor-2 up-regulation by Fc-receptor activation in human neutrophils. FASEB J. 2010;24(6):2116-25. http://doi.org/10.1096/fj.09-146167

27. Lan RS, Stewart GA, Goldie RG, Henry PJ. Altered expression and in vivo lung function of protease- activated receptors during influenza A virus infection in mice. Am J Physiol Lung Cell Mol Physiol. 2004;286(2):388L-98L. http://doi.org/10.1152/ajplung.00286.2003

28. Yang YL, Serrano MG, Sheoran AS, Manque PA, Buck GA, Widmer G. Over-expression and localization of a host protein on the membrane of Cryptosporidium parvum infected epithelial cells. Mol Biochem Parasitol. 2009;168(1):95-101. http://doi.org/10.1016/j.molbiopara.2009.07.004 